\title{
Significance of Cohen's Class for Time Frequency Analysis of Signals
}

\author{
Azeemsha Thacham Poyil \\ College of Computers and \\ Information Technology \\ Taif University, Saudi Arabia
}

\author{
Sultan Aljahdali \\ College of Computers and \\ Information Technology \\ Taif University, Saudi Arabia
}

\author{
Nasimudeen.K.M \\ College of Computers and \\ Information Technology \\ Taif University, Saudi Arabia
}

\begin{abstract}
In this paper, a study of quadratic transformations under Cohen's class is presented, to see the variations in resolution for performing time-frequency analysis of signals. The study concentrated on the analysis of linear chirp signals and nonstationary signals in presence of noise as well as without noise. The resolutions based on Wavelet Transform, Short Time Fourier Transform are analysed. The effects of widow length, wavelet scale and presence of noise are researched and analyzed against the performance of different time-frequency representations. The Cohen's class is a class of time-frequency quadratic energy distributions which are covariant by translations in time and in frequency. This important property by the members of Cohen's class makes those representations suitable for the analysis and detection of linear as well as transient signals. Spectrogram, the squared modulus of Short Time Fourier Transform is considered to be an element of Cohen's class since it is quadratic and also co-variant in time and frequency. Wigner Ville Distribution is another member of Cohen's class which can be extended to many other variants by changing the kernel functions used for cross-term reductions. The trade-off in the time-frequency localization are studied and demonstrated with the help of different plots. The result of this study can be applied to enhance the detection and analysis of signals and to develop efficient algorithms in medical diagnosis as well as defense applications.
\end{abstract}

\section{Keywords}

Wavelet Transform (WT), Scalogram, Short Time Fourier Transform (STFT), Fast Fourier Transform (FFT), Wigner Ville Distribution (WVD), Cohen's Class, Spectrogram

\section{INTRODUCTION}

The use of time-frequency techniques in signal analysis and detection has been studied by many researchers at times. The major reason for adopting these techniques in medical and defense fields are the amount of simultaneous information we get from this. In this paper a study of quadratic transformations under Cohen's class is presented, to see the variations in resolution for performing time-frequency analysis of signals. The Cohen's class is a class of timefrequency quadratic energy distributions which are covariant by translations in time and in frequency ${ }^{[1]}$. The extraction of useful data from a noisy multi-component signal is always a big challenge for the researchers in the field of signal processing. The concentration was on the study of linear chirp signals and non-stationary signals using Wavelet Transform, Scalogram, Spectrogram, STFT and Wigner Ville. For this purpose many built-in MATLAB functions provided by the Time-Frequency Toolbox have been used ${ }^{[1]}$. To start with, some generic definitions and methods in time-frequency signal analysis are described. After that, the need of timefrequency representations is explained with the help of examples. To the end of this paper, the results of many transformations are plotted and explained for different types and combinations of input signals.

\section{BACKGROUND AND RELATED WORK}

\subsection{STFT and Spectrogram}

Short Time Fourier Transform is calculated for a signal by pre-windowing the signal $\mathrm{s}(\mathrm{t})$ around a particular time $\mathrm{t}$ and then calculating the Fourier transform ${ }^{[1]}$. And this is repeated for all time instants $t$ as in the equation 1.

$$
\operatorname{STFT}_{x}(t, v)=\int_{-\infty}^{+\infty} s(u) h(u-t) e^{-j 2 \pi v u} d u
$$

Here $\mathrm{h}$ is the window function. The Spectrogram is defined as the squared modulus of STFT and is by nature a representation of the signal energy.

$$
S_{x}(t, v)=\left|\int_{-\infty}^{+\infty} s(u) h^{*}(u-t) e^{-j 2 \pi v u} d u\right|^{2}
$$

\subsection{Wavelet Transform and Scalogram}

A continuous wavelet transform (CWT) is calculated by projecting a signal $s(t)$ on a family of zero-mean functions called wavelets. The wavelets are deduced from a basic function called mother wavelet by translations and dilations [1]

$$
T_{x}(t, a ; \Psi)=\int_{-\infty}^{+\infty} s(u) \cdot \Psi_{t, a}^{*}(u) d u
$$

Where

$$
\Psi_{t, a}(u)=|a|^{-1 / 2} \cdot \Psi\left(\frac{u-t}{a}\right)
$$

The variable ' $a$ ' is called scale factor. If the value of $|a|$ is greater than 1 , in it dilates the wavelet and if the value of $|\mathrm{a}|$ is less than 1 it compresses the wavelet. The major difference between wavelet transform and STFT is that, when the scale factor is changed, then both the duration and the bandwidth of the wavelet are changed. But the shape of wavelet will be the 
same as before. Another difference is that the WT uses short windows at high frequencies and long windows at low frequencies ${ }^{[1]}$

Scalogram of a signal $\mathrm{s}(\mathrm{t})$ is defined as the squared modulus of the Continuous Wavelet Transform, which describes the energy of the signal in time-scale plane.

\subsection{Wigner Ville Distribution}

Wigner Ville Distribution (WVD) is a bilinear function of the signal calculated using the formula,

$$
W V D_{s}(t, v)=\int_{-\infty}^{+\infty} \mathrm{s}(\mathrm{t}+\tau / 2) . \mathrm{s} *(\mathrm{t}-\tau / 2) \cdot e^{-j 2 \pi \nu \tau} \cdot d \tau
$$

Where $t$ represents time and $v$ represents frequency. One major difference between the WVD and STFT is that the calculation of WVD does not make use of any windows ${ }^{[1],[2]}$.

\subsection{Time and Frequency Marginal}

A joint time and frequency energy density $\rho_{x}(t, v)$ is defined in terms of the signal energy as

$$
E_{x}=\int_{-\infty}^{+\infty} \int_{-\infty}^{+\infty} \rho_{x}(t, v) d t, d v
$$

Since the energy is a quadratic function of the signal, the time-frequency energy distributions will also be in general quadratic representations. The energy density parameter also satisfies marginal properties as define below. Integrating the energy density along one time axis will give rise to the energy density corresponding to frequency and vice versa ${ }^{[1]}$.

$$
\begin{aligned}
& \int_{-\infty}^{+\infty} \rho_{x}(t, v) d t=|X(v)|^{2} \\
& \int_{-\infty}^{+\infty} \rho_{x}(t, v) d v=|x(t)|^{2}
\end{aligned}
$$

Using the generic mathematical equations (7) and (8) for quadratic distributions, the marginal of WVD along frequency axis can be described by

$$
\begin{aligned}
& \int_{-\infty}^{+\infty} W_{x}(t, v) d v=|x(t)|^{2} \\
& \int_{-\infty}^{+\infty} W_{x}(t, v) d t=|X(v)|^{2}
\end{aligned}
$$

\subsection{Cohen's Class}

Cohen's class of signals are generally represented by

$$
C_{s}(t, v ; \Pi)=\int_{-\infty}^{+\infty} \int_{-\infty}^{+\infty} \Pi(\mathrm{s}-\mathrm{t}, \xi-v) \cdot \mathrm{W}_{\mathrm{x}}(\mathrm{s}, \xi) \cdot \mathrm{ds} \cdot \mathrm{d} \xi
$$

where, $\Pi$ is known as smoothing function. So, the Cohen's class can be defined as a smoothed version of the WVD. By properly selecting the smoothing function we can create many variants of WVD.

The spectrogram is an element of the Cohen's class since it is quadratic, covariant with time and frequency, and also preserves energy [1]. Spectrogram can be represented as a smoothed version of WVD by selecting the smoothing function as the 'WVD of the window' function $h$.

As mentioned before, WVD is an element of Cohen's class which can be understood by selecting the smoothing function as a double Dirac function. The interference terms in WVD can be effectively removed by selecting a suitable smoothing kernel. There are many variants possible for WVD, for example Smoothed WVD and Smoothed Pseudo WVD. Each of them is implemented by selecting suitable smoothing functions.

The pseudo WVD is defined as the frequency smoothed version of the WVD according to the equation,

$$
P W V D_{x}(t, v)=\int_{-\infty}^{+\infty} H(v-\xi) \cdot \mathrm{W}_{\mathrm{x}}(\mathrm{t}, \xi) \cdot \mathrm{d} \xi
$$

Here, $H(v-\xi)$ is the Fourier transform of smoothing window $h(t)$. Here a compromise is done on many good features of WVD in order to smooth out the cross terms.

Smoothed Pseudo WVD (SPWVD) is another variant in Cohen's class which is implemented by splitting up the smoothing function so as to provide an independent smoothing in time domain as well as frequency domain. The smoothing function can be represented as

$$
\Pi(\mathrm{t}, v)=\mathrm{g}(\mathrm{t}) \cdot \mathrm{H}(-v)
$$

\subsection{Time-Frequency resolution}

The need of simultaneous time-frequency localizations can be understood using the below example. Figure 1 shows the Gaussian modulated linear FM signal with an analytic complex Gaussian noise with mean 0 and variance 1 added to it. The FFT spectrum of the signal is also seen in the figure. It is seen that the visibility of the signal spectrum is affected due to the presence of noise. In order to map the time and frequency information easily we can also look at the STFT plot of the signal in the same figure.
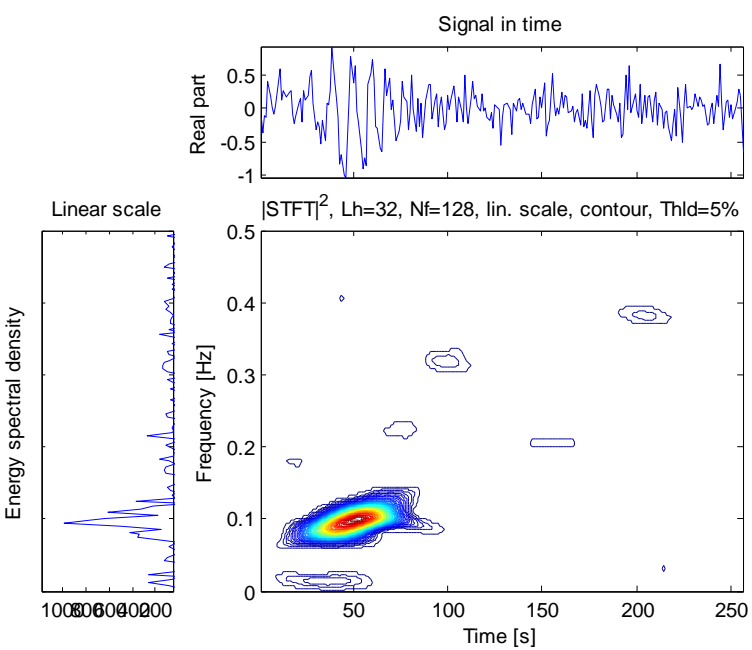

Fig 1: STFT of the Noisy Signal

In the Figure 1 the wide colored area in the contour plot of the signal's STFT represents the major signal component, and some noise components spread away from that. It can be noticed that the information we get from this plot helps us to map between the frequency content and the time content of the signal in the same plot. We can state which frequency component exist at which point of time.

So it can be understood that the time-frequency representation helps us to easily study and understand the frequency contents of a signal at different time instants. Many researchers worked in this area have produced lots of useful results towards 
improving and comparing the performance. For example, N. Zaric et al, did the implementation of a robust time-frequency distribution for analysis of signals in presence of noise. This included the development of an L-estimate STFT which uses a sorting operation ${ }^{[3]}$. Another work by G. Yu proposed a work on audio de-noising by using threshold in timefrequency block ${ }^{[4]}$. Y. S. Wei and team researched on algorithms for eliminating interference between signals and thus helping multi-target signals in high frequency Radars ${ }^{[5]}$. Y.C.Jiang worked on generalized time-frequency distributions for multi-component polynomial phase signals. Three algorithms are proposed for removing the interference terms in multi-component signals through the generalized time-frequency distributions ${ }^{[6]}$. Yictor Sucic and team did another study on optimization algorithm for selecting quadratic Time-frequency Distributions in order to select the distribution which provides the best localization of signal components [7]. Daniel Mark Rosser researched on timefrequency analysis of a carrier signal with additive white Gaussian noise in order to help in signal detection problems, which is addressed by time-frequency processing of the signal ${ }^{[8]}$. Boualem Boashash have extensively worked on timefrequency signal analysis and processing proposing many algorithms ${ }^{[9]}$. Higher order time-frequency poly-spectra are studied by Alfred Hanssen and team ${ }^{[10]}$. Juan D MartinezVargas et al have worked on time-frequency based algorithms for feature extraction of transient bio-signals ${ }^{[11]}$. In another research by Ervin Sejdic and associates, the time-frequency features are studied by considering the energy concentration as the parameter of analysis ${ }^{[12]}$. H. Zou et al had worked on parametric time-frequency representations using translated and dilated windowed exponential FM functions [13]. Hongxing Zou and team also worked on a similar area of time-frequency distributions for parametric TFRs using special class of transformation group called as 'semi-affine' transformation group. This approach helps in achieving a good visibility for highly non-stationary signals ${ }^{[14]}$. Jun Jason Zhang et al proposed algorithms for time-frequency characterization of signals in order to help in receiver waveform design for shallow water environments [16]. Another research by B. Zhang and team discusses about the time-frequency distribution of Cohen's class with a compound kernel and its application in speech signal processing ${ }^{[17]}$.

\section{METHOD FOR ANALYSIS}

For the study, many types and combinations of signals are considered. As the first step, the representation of linear chirp signals with and without the presence of noise was studied. The WVD of the signal is calculated using the formula (5). In the second step transient signals are studied with and without the presence of noise. In the third step a LFM signal is undergone rectangular amplitude modulation and then, the STFT and Spectrogram are studied for different window functions. The Wavelet Transform and Scalogram are studied for 'Altes Signal' in the next step. The time-frequency resolution is compared against that achieved by using WVD. To the end of the research, the cross-terms and time-frequency localizations achieved using 3 variants of Cohen's class are studied and compared.

\section{RESULTS}

The above methods are implemented in MATLAB using the functions provided by Time Frequency Toolbox. After applying the algorithms for quadratic representation of different signals, a set of results as described are obtained.
The time and frequency information of a linear chirp signal are represented independently in Figure 2 and Figure 3. The WVD of the same signal is also shown in Figure 4. A straight line plot can be seen in the time-frequency domain. We can also note the good localization provided by WVD in this case.

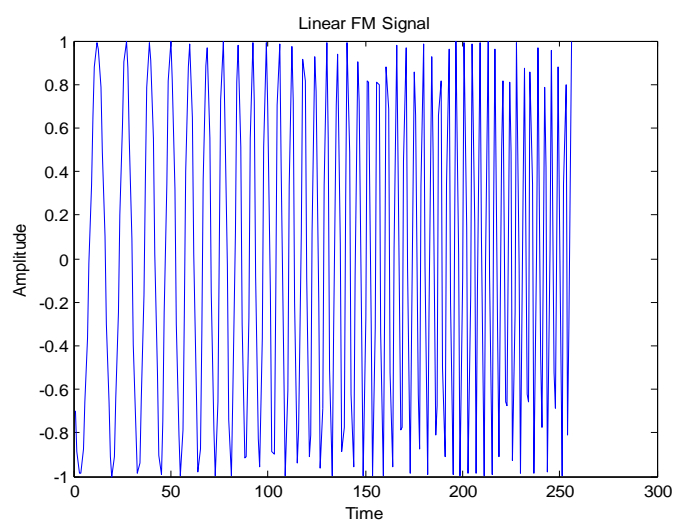

Fig 2: Linear Chirp Signal

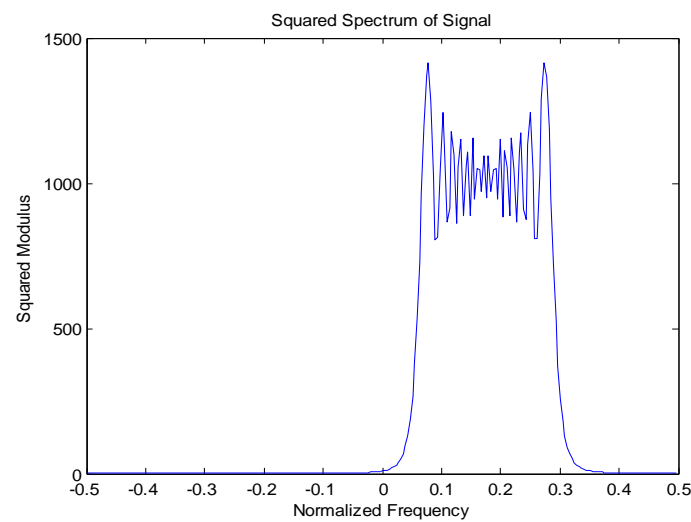

Fig 3: Signal Spectrum of the LFM Signal

Figure 5 shows another analysis of the same signal in presence of noise. The spectrum of the noisy signal is not informative in terms of the actual and noise signal. But the WVD plot in Figure 5 shows a clear representation of the LFM signal even in the presence of noise. A line can be seen that represents the linear chirp clearly.

\subsection{STFT and Spectrogram}

A transient signal in presence of noise is generated and analyzed using STFT and Spectrogram as in Figure 8. The transient signal is generated by multiplying an amplitude modulation signal and a frequency modulation signal. Later and analytic complex Gaussian noise signal is added to it as in Figure 6 and Figure 7. The corresponding time and frequency diagrams show almost unidentifiable signal details which don't seem to be useful for a good analysis. On the other hand the spectrogram representation as in Figure 8 gives a good about the simultaneous time and frequency elements. The concentrated area in the middle of the plot represents the presence of the signal even in an environment of noise.

In the next step, the performance of Spectrogram is analyzed using another input signal generated by a linear frequency modulation multiplied with a rectangular amplitude modulation. Different types of window functions were 
selected for the calculation of STFT. When a dirac impulse was used as the window function a good time resolution and a poor frequency resolution was obtained.

Signal in time
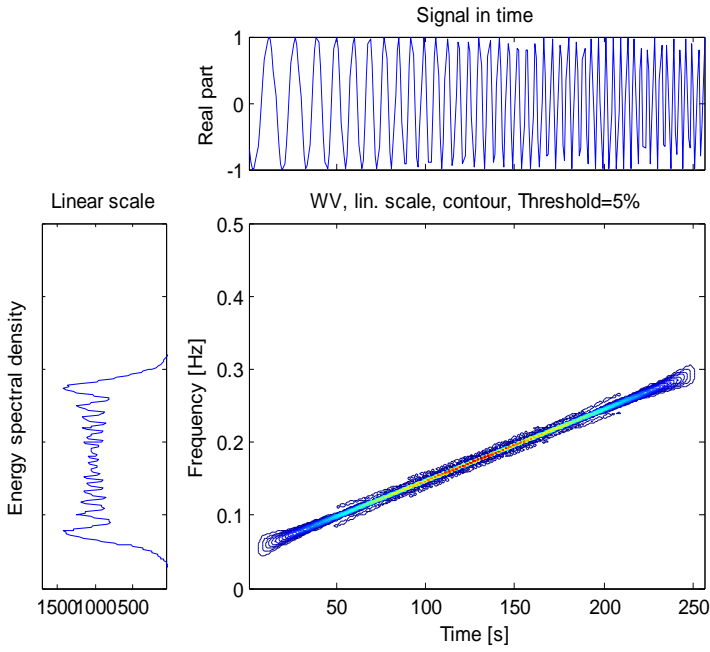

Fig 4: WVD of the LFM Signal
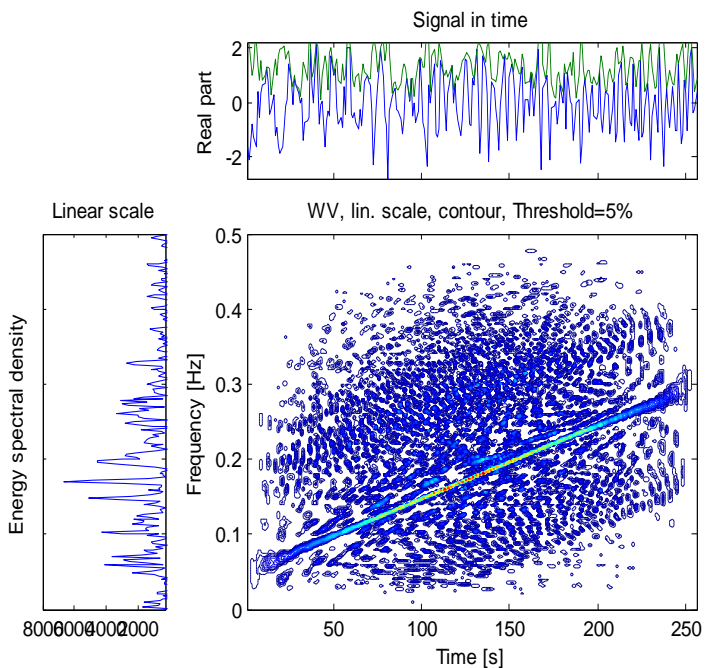

Fig 5: WVD of the Signal with Noise

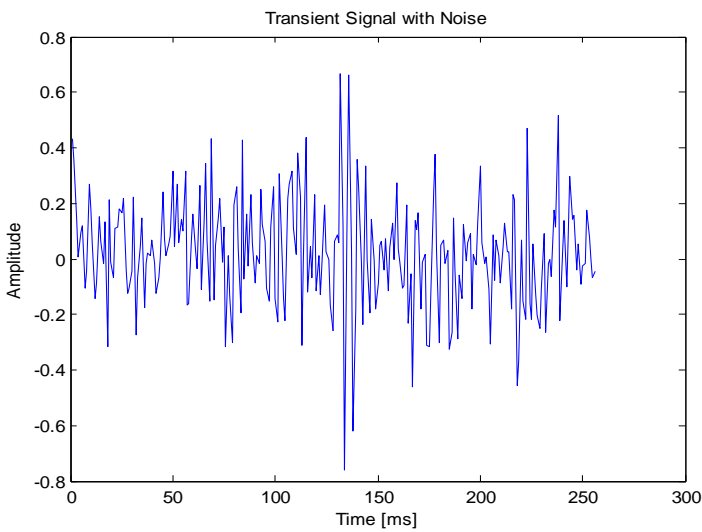

Fig 6: Transient Signal with Noise

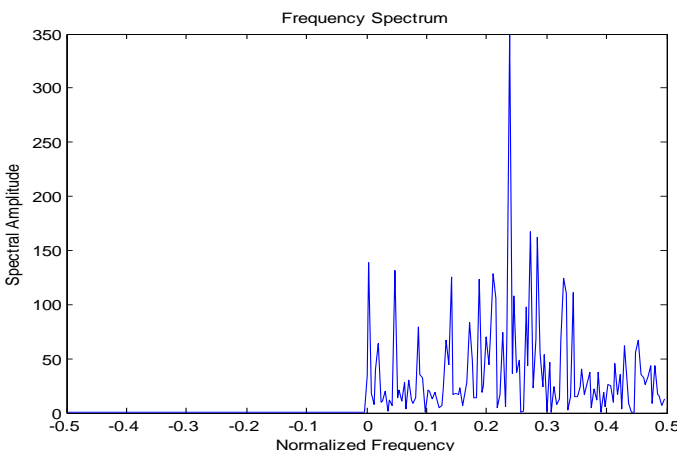

Fig 7: Signal Spectrum

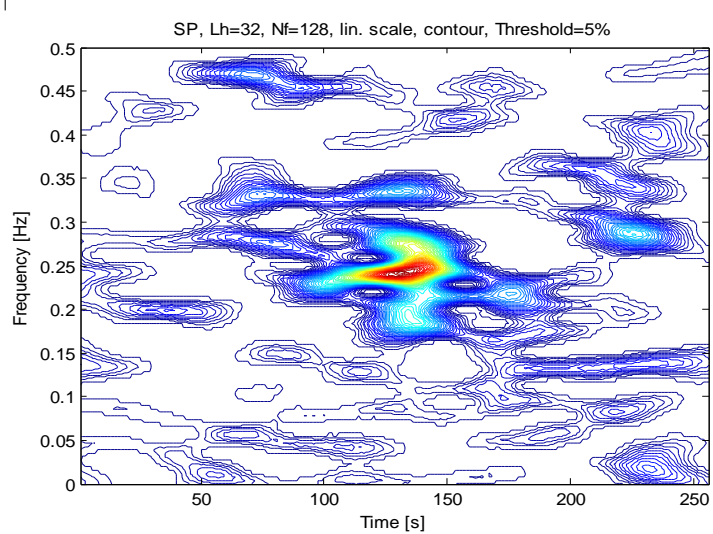

Fig 8: Spectrogram of the transient Signal with Noise

The plot for Spectrogram using a dirac impulse as the window function is as shown in Figure 9.

Later a good frequency resolution and a poor time resolution was achieved by analyzing the same signal using STFT with a constant window as the window function. The plot for Spectrogram using a constant window function is as timing window is shown in Figure 10.
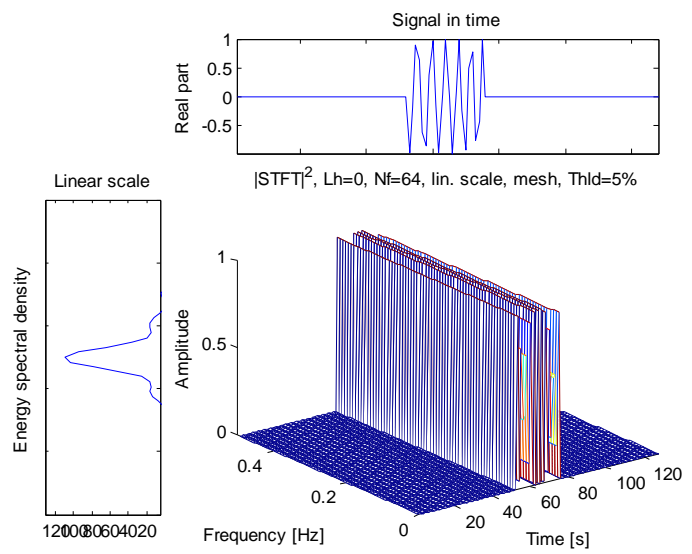

Fig 9: Spectrogram of the Signal using a Dirac Window Function 


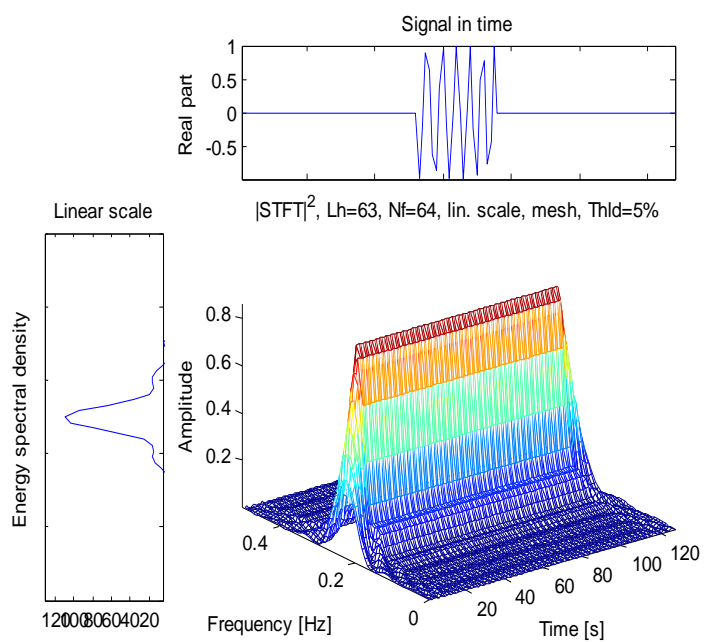

Fig 10: Spectrogram of the Signal using a Constant Window Function

In a next step of analysis, the effect of using a short window function and a long widow function for calculating spectrogram is demonstrated. Spectrogram of two linear chirp signals is implemented. A Gaussian function of length 11 is used to perform the windowing. We can notice a good time resolution with compromise on frequency resolution as in Figure 11. In a second study for the same signal, a long Gaussian window function of length 127 is used to calculate the Spectrogram. A good frequency resolution was observed with compromise on the time resolution as visible in the Figure 12. It can also be seen that the interference terms affect the readability of 2 chirp signals in both of the above cases.

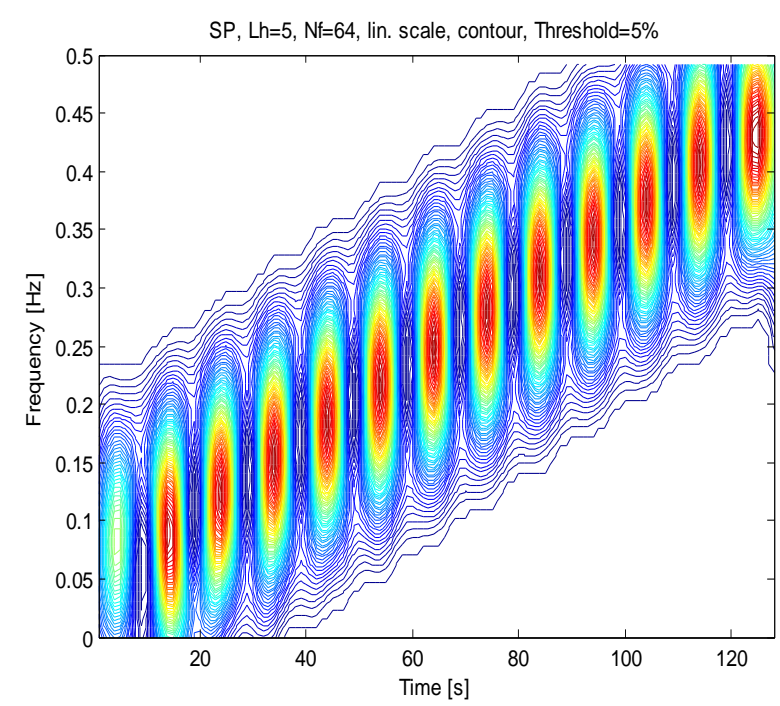

Fig 11: Spectrogram of 2 LFS Signals Using a Short Gaussian Window (Length 11)

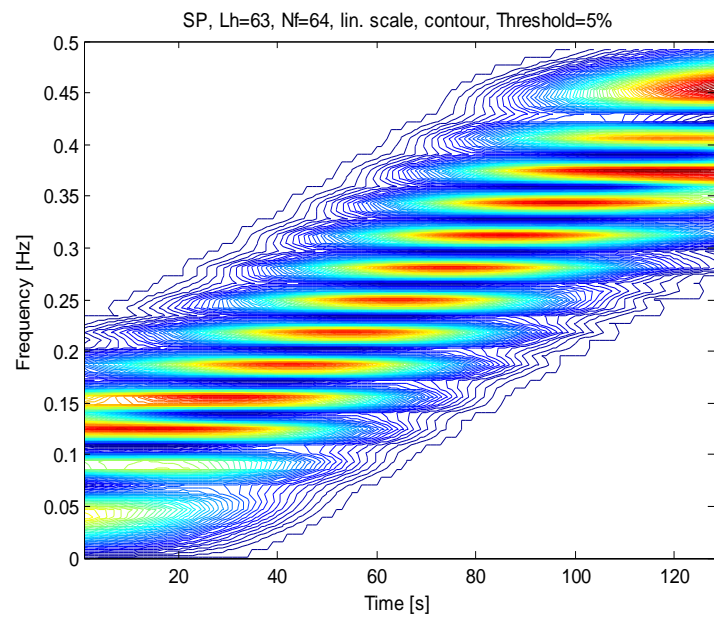

Fig 12: Spectrogram of 2 LFS Signals Using a Long Gaussian Window (Length 127)

\subsection{Scalogram and Wigner Ville Distribution}

The time-frequency localization of many signals is also compared by using Wavelet Transform and Wigner-Ville distribution. In the first study, the Scalogram (squared modulus of Continuous Wavelet Transform) is calculated using Morlet wavelet for an input signal containing 24 points of an "Altes Signal" of 256 samples. Later for the same signal, the Wigner Ville Distribution is also calculated. The input signal in time domain is as shown in Figure 13. Figure 14 shows the Scalogram representation of the signal and Figure 15, the WVD. The contour plots of the Scalogram and WVD show a huge difference in the resolution. The WVD shows a narrower plot in comparison with the Scalogram, which clearly states the advantage of WVD.

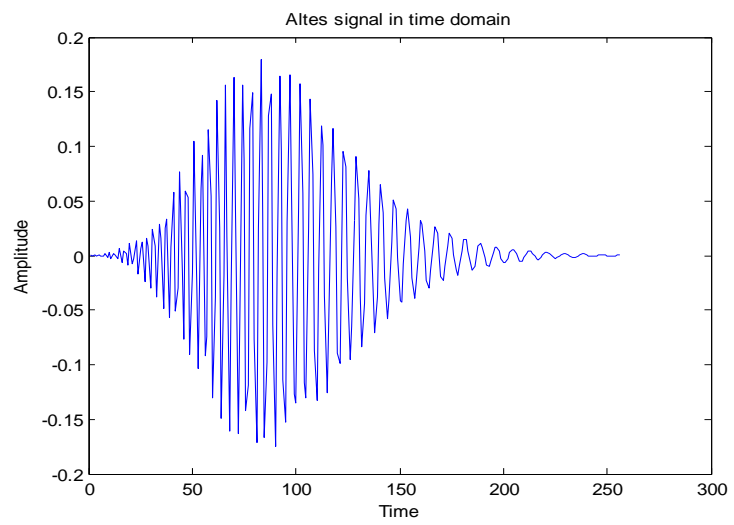

Fig 13: Altes Signal” of 256 samples 


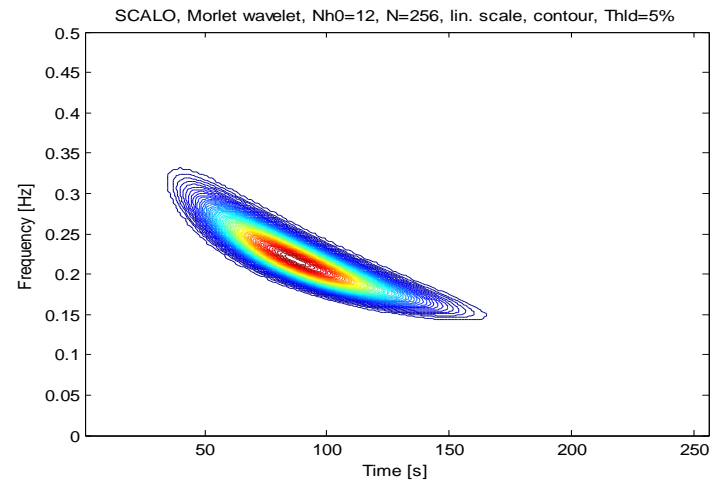

Fig 14: Scalogram of the Signal

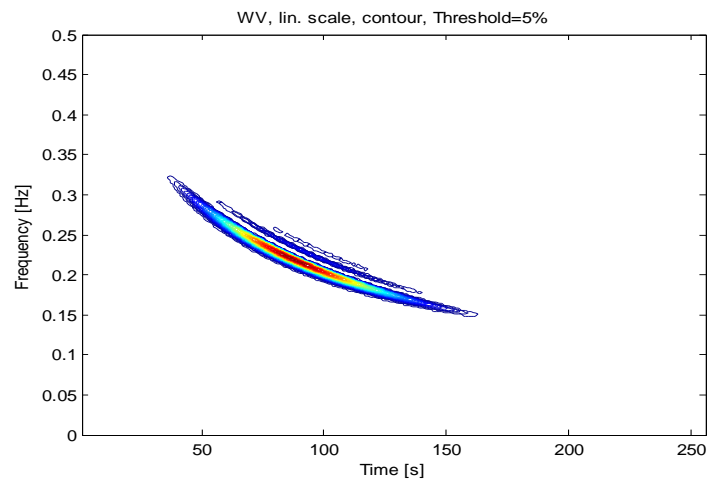

Fig 15: WVD of the Signal

In a next example, the sum of two constant FM signals is studied with different frequencies, to compare the timefrequency resolutions between scalogram and WVD variants in Cohen's class. Two constant FM signals are considered with normalized frequencies 0.15 and 0.35 respectively. Along with the Scalogram analysis, the different variants of Cohen's Class are also applied in order to study the difference in localizations. This includes the WVD, Pseudo WVD and Smoothed Pseudo WVD. The scalogram of the signal as shown in Figure 16 does not give a good resolution as given by the WVD of the signal as in Figure 17. But in comparison to WVD, the cross terms are not present in Scalogram

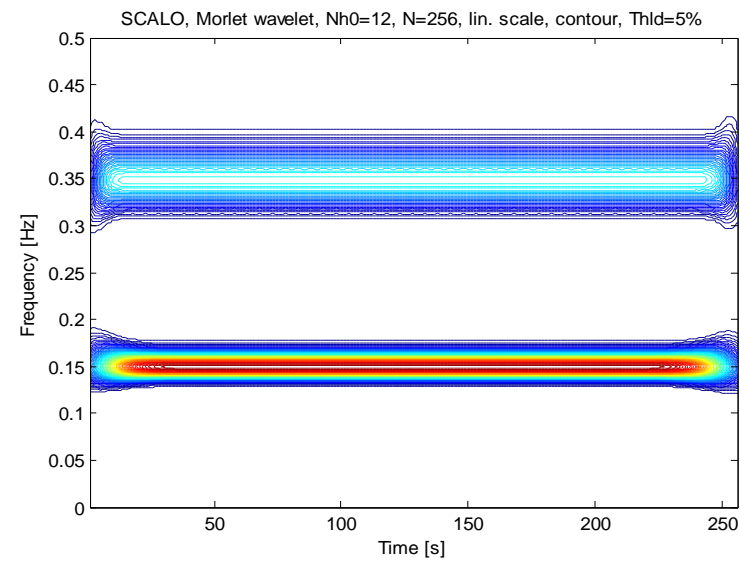

Fig 16: Scalogram of the Signal

Please note the interference terms present in the middle of two constant signals as in the plot of WVD in Figure 17.
Now let us see the result of applying the Pseudo WVD by smoothing in frequency domain. As we can see in Figure 18, this doesn't really provide a reduction in cross-terms; instead it results in a reduction in frequency resolution.

The Figure 19 shows the Smoothed Pseudo WVD of the same signal. The SPWVD does an independent smoothing in time as well as in frequency domain, which results in a reduction in cross terms also. When we compare the results of all the above three variants of Cohen's class, it can be understood that the Smoothed Pseudo WVD of the signal is a better one in terms of the time-frequency resolution and cross-term reduction.

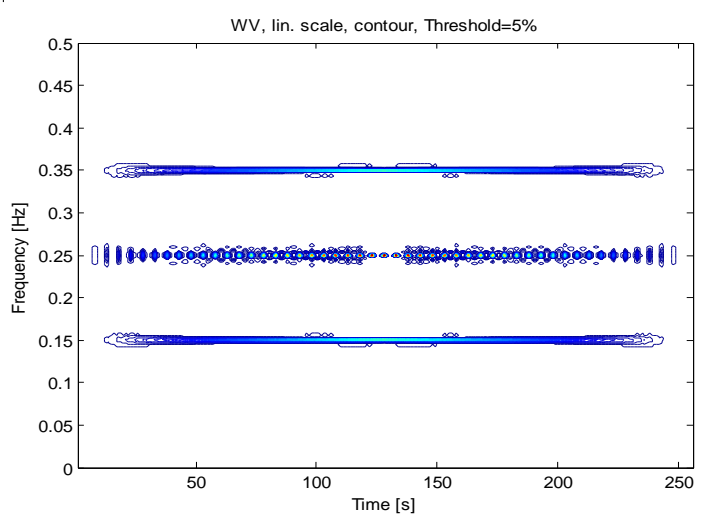

Fig 17: WVD of the Signal

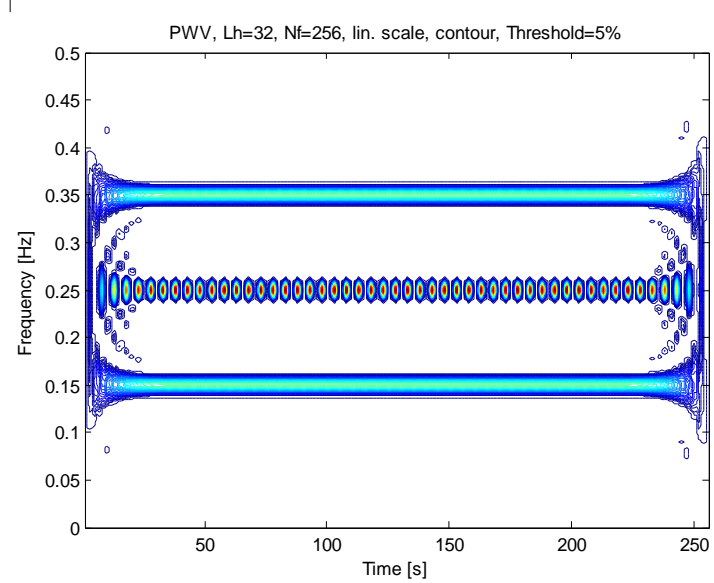

Fig 18: Pseudo WVD of the Signal

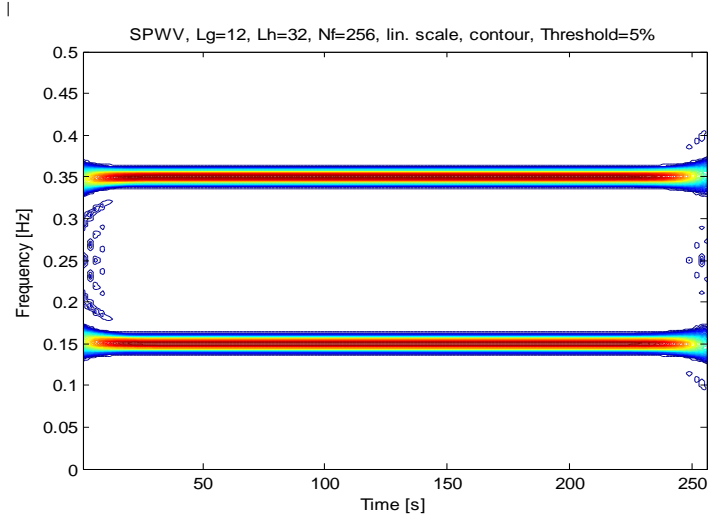

Fig 19: Smoothed Pseudo WVD of the Signal 
In the next analysis, a combination of one linear FM signals with a constant FM signal of normalized frequency 0.35 is used. The scalogram representation as in Figure 20 doesn't create any cross-terms, but at the same time doesn't give us a good time-frequency resolution. On the other hand the WVD as in Figure 21, does give a good resolution but with presence of cross-terms. As before, the Pseudo WVD as in Figure 22 neither improves the cross-terms nor the resolution.

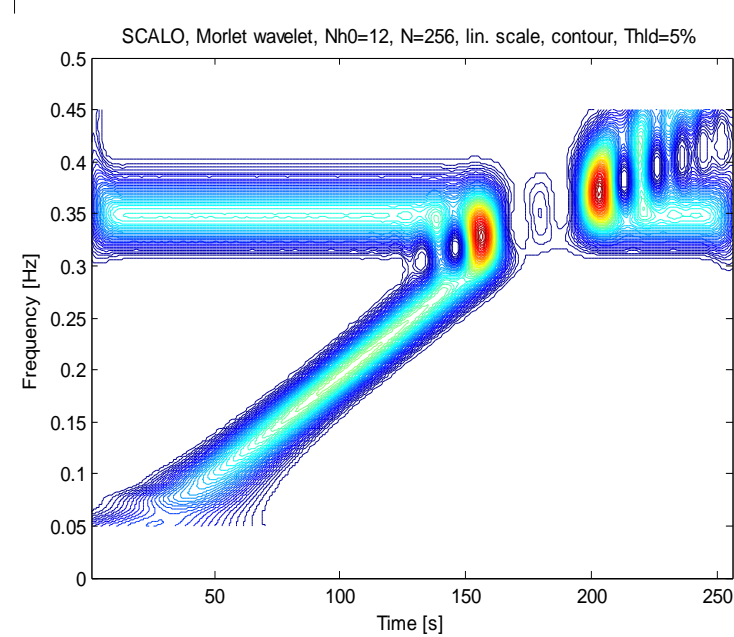

Fig 20: Scalogram of the Signal

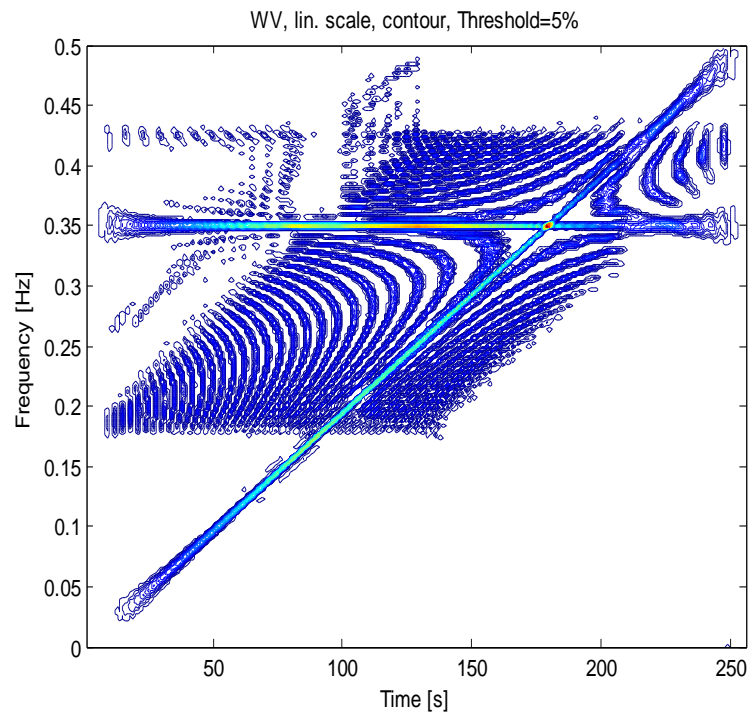

Fig 21: WVD of the Signal

Here again, the Smoothed Pseudo WVD as in Figure 23 presents a good compromise on the resolution against the cross-term reduction.

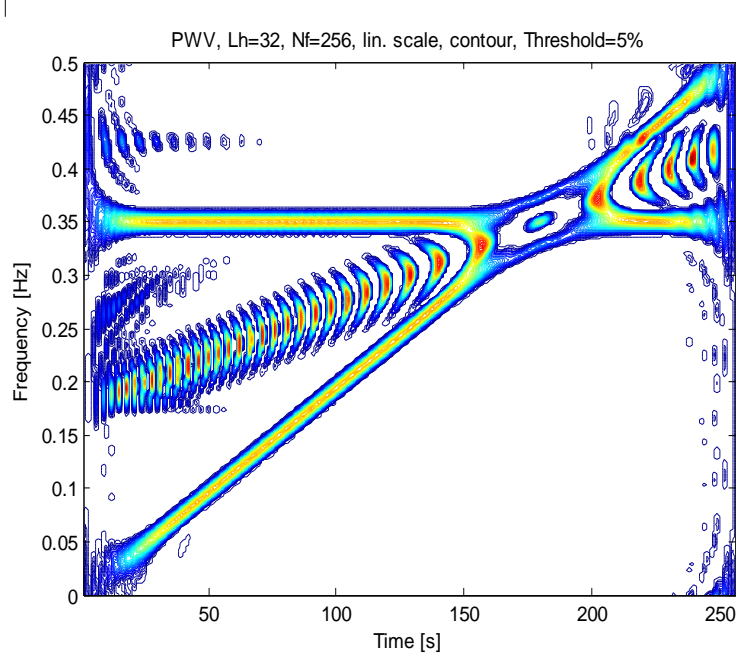

Fig 22: Pseudo WVD of the Signal

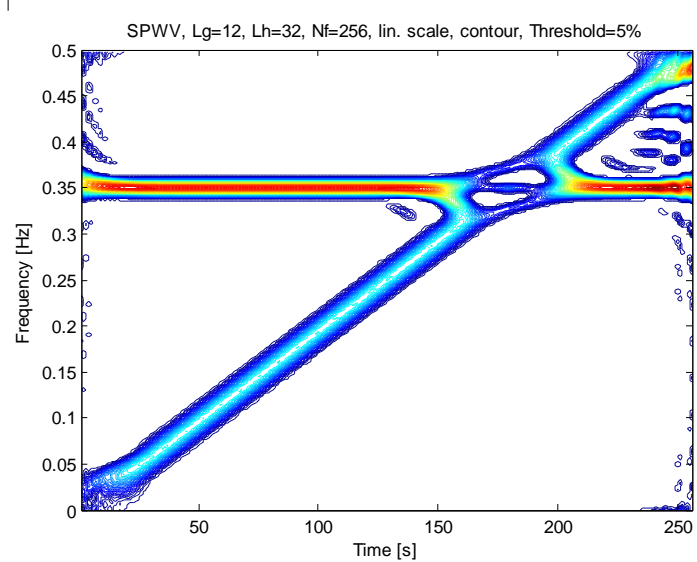

Fig 23: Smoothed Pseudo WVD of the Signal

\section{CONCLUSION}

From the above experiments and results, it can be understood that, the different variants of the Cohen's class exhibit variations in performance during signal analysis and detection. In case of multi-component signals, the mathematical interference terms are to be compromised against the signal resolution in time - frequency domain. If we are least bothered about the presence of cross terms, and are looking only for good time-frequency localization, WVD is found to be a good option. Among the different representations that are implemented using Wavelet Transform, Spectrogram, Wigner Ville, Pseudo Wigner Ville and Smoothed Pseudo Wigner Ville, it can be concluded that the Smoothed Pseudo WVD gives a good compromise on the signal resolution against the cross-terms. As a future work, many new and effective variants of WVD can be developed by developing suitable kernel functions. And further studies can be conducted in order to assess the improvements in the time-frequency localization. 


\section{ACKNOWLEDGEMENT}

The authors gratefully acknowledge the directions from the MATLAB Time Frequency Toolbox for providing different functions in order to perform the time-frequency transformations, and thus helping the successful completion of this research paper.

\section{REFERENCES}

[1] François Auger, Patrick Flandrin, Paulo Gonçalvès, Olivier Lemoine, Tutorial - Time Frequency Toolbox for use with MATLAB, 1996

[2] Azeemsha Thacham Poyil, Shadiya Alingal Meethal, "Cross-term Reduction Using Wigner Hough Transform and Back Estimation", IEEE Explore, Issue date 23-25 Aug. 2012

[3] N. Zaric, N. Lekic, and S. Stankovic, "An implementation of the L-estimate distributions for analysis of signals in heavy-tailed noise," IEEE Transactions on Circuits and Systems II, Vol. 58, No. 7, July 2011, pp. 427-431

[4] G. Yu; S. Mallat, and E. Bacry, "Audio denoising by time-frequency block thresholding," IEEE Transactions on Signal Processing, Vol. 56, No. 5, May 2008, pp. 1830-1839.

[5] Y. S. Wei and S. S. Tan, "Signal decomposition of HF radar maneuvering targets by using S2-method with clutter rejection,"Journal of Systems Engineering and Electronics, Vol. 23, No. 2, April 2012, pp. 167 - 172.

[6] Y.C.Jiang, "Generalized time-frequency distributions for multi-component polynomial phase signals,"Signal Processing, Vol. 88, No. 4, April 2008, pp. 984-1001.

[7] Yictor Sucic and Boualem Boashash, "Optimization Algorithm for Selecting Quadratic Time-frequency Distributions: Performance Results and Calibration" International Symposium on Signal Processing and its Applications (ISSPA), Kuala Lumpur, Malaysia, 2001

[8] Daniel Mark Rosser, "Time-Frequency Analysis of a Noisy Career Signal", Naval Post Graduate School Monterey, California, 1996

[9] Boualem Boashash, "Time-Frequency Signal Analysis and Processing - A Comprehensive Reference", Queensland University of Technology, Brisbane, Australia, 2003

[10] Alfred Hanssen, and Louis L. Scharf, "A Theory of Polyspectra for Nonstationary Stochastic Processes", IEEE Transactions On Signal Processing, Vol. 51, No. 5, 2003

[11] Juan D Mart'inez-Vargas, Juan I Godino-Llorente, and German Castellanos-Dominguez, "Time-frequency based feature selection for discrimination of nonstationary biosignals", EURASIP 2012

[12] Ervin Sejdic, Igor Djurovic and Jin Jiang, "TimeFrequency Feature Representation Using Energy Concentration: An Overview of Recent Advances", ScienceDirect, Digital Signal Processing 19 (2009) 153183
[13] H. Zou, Q. Dai, R. Wang, and Y. Li, "Parametric TFR via windowed exponential frequency modulated atoms," IEEE Signal Processing Letters, Vol. 8, No. 5, May 2001,

[14] Hongxing Zou, Dianjun Wang, Xianda Zhang, Yanda Li, "Nonnegative time-frequency distributions for parametric time-frequency representations using semiaffine transformation group", Signal Processing 85 (2005)

[15] Time-series analysis in marine science and applications for industry, 17- 21 September 2012 - LogonnaDaoulas, France

[16] Jun Jason Zhang, Antonia Papandreou-Suppappola, Bertrand Gottin, and Cornel Ioana, "Time-Frequency Characterization and Receiver Waveform Design for Shallow Water Environments", IEEE Transactions On Signal Processing, Vol. 57, No. 8, August 2009

[17] B. Zhang and S. Sato, "A time-frequency distribution of Cohen's class with a compound kernel and its application to speech signal processing," IEEE Transactions on Signal Processing, Vol. 42, No. 1, Jan. 1994, pp. 54-64.

Azeemsha Thacham Poyil, M Tech. He was born in Kerala, India in 1979. He received his Bachelor degree in Electronics and Communication Engineering from Kannur University, India in 2001. Later he received his Master degree in electronics from Cochin University of Science and Technology, India in 2005. He is currently a Lecturer in College of Computers and Information Technology, Taif University, Saudi Arabia. He was previously working as specialist software engineer with Bosch Thermo Technology, Germany and Robert Bosch Engineering and Business Solutions, India in the industrial product development. His major research areas are Digital Signal Processing and Embedded Systems.

Sultan Hamadi Aljahdali, $\mathrm{PhD}$. He is graduated with $\mathrm{Ph} . \mathrm{D}$, in Information Technology from George Mason University, USA in 2003. He received his B.S from Winona State University and M.S. with honor from Minnesota State University in Mankato. He is currently an associate professor in Dept. of Computer Science, Taif University. He is also the dean of college of Computer \& Information Technology, Taif University Saudi Arabia. His research interests include Performance Prediction in Software Engineering, Software Modeling and Cost Estimation, Performance Modeling.

Nasimudeen.K.M, M.E (CS), M.C.A. He was born in Kerala, India in 1979. He received his Bachelor degree in Electronics from Kerala University, India in 2000. Later he received his M.C.A(Master of Computer Application) degree from Madurai Kamaraj University, Madurai, India in 2003 and he obtained his M.E(Master of Engineering) degree in computer science and Engineering from Anna University, Chennai in 2005. Pursuing his $\mathrm{PhD}$ in computer science and Engineering from Anna University Chennai, India. Presently he is working as a Lecturer in College of Computers and Information Technology, Taif University, Taif, Kingdom of Saudi Arabia. He was previously worked as Lecturer/HOD of Computer Science department of Aalim Mohammed Salegh College of Engineering, Chennai, India. His major research areas are Natural language Processing and Neural network. 